\title{
PROPIEDADES ENERGÉTICAS DE LA HARINA DE MACA (Lepidium peruvianum Chacón o Lepidium meyenii Walpers)
}

\author{
Violeta Romero*a, Arminda Tirado ${ }^{\text {a }}$, Mónica Durán ${ }^{\mathrm{b}}$, Juan Z. Dávalos ${ }^{\mathrm{b}}$
}

\section{RESUMEN}

En este trabajo se estudiaron propiedades termofísicas y termoquímicas de la harina de maca comercial seca, utilizando calorimetría de combustión y calorimetría diferencial de barrido (DSC). Se determinó el valor calorífico neto o energía másica de combustión (419 \pm $3 \mathrm{Kcal} / 100 \mathrm{~g}$ ), calores específicos (entre -4 y $70 \mathrm{oC}$ ) en el que se registra para $25^{\circ} \mathrm{C}$ el c.e $=$ $1.46 \pm 0.03 \mathrm{~J} / \mathrm{g} . \mathrm{oC}$; y parámetros térmicos, como la temperatura y entalpía, tanto del proceso de descomposición $\left(T_{\max }=210.1 \pm 0.4 \mathrm{oC} \mathrm{y} \Delta H_{\text {dec }}=82 \pm 10 \mathrm{~J} / \mathrm{g}\right)$ como de la gelatinización $\left(T_{p}=76.9 \pm 0.4 \mathrm{oC} ; \Delta H_{g e l}=5.6 \pm 0.5 \mathrm{~J} / \mathrm{g}\right)$.

Palabras clave: calor específico, energía de descomposición, harina de maca, gelatinización de la maca.

\section{ENERGETIC PROPERTIES OF MACA (Lepidium peruvianum Chacón or Lepidium meyenii Walpers) FLOUR}

\begin{abstract}
In this work thermophysical and thermochemical properties of dried commercial maca flour were studied by combustion calorimetry and differential scanning calorimetry (DSC). Net calorific value or combustion massic energy $(419 \pm 3 \mathrm{Kcal} / 100 \mathrm{~g})$; specific heats (between -4 and $70{ }^{\circ} \mathrm{C}$ ) where c.e. for $25^{\circ} \mathrm{C}$ is $1.46 \pm 0.03 \mathrm{~J} / \mathrm{g} .{ }^{\circ} \mathrm{C}$; and thermal parameters, such as temperature and enthalpy, of decomposition $\left(T_{\max }=210.1 \pm 0.4{ }^{\circ} \mathrm{C}, \Delta H_{\text {dec }} 82 \pm 10 \mathrm{~J} / \mathrm{g}\right)$ and gelatinization $\left(T_{p}=76.9 \pm 0.4{ }^{\circ} \mathrm{C}, \Delta H_{\text {gel }}=5.6 \pm 0.5 \mathrm{~J} / \mathrm{g}\right)$ processes were evaluated.
\end{abstract}

Key words: specific heat, decomposition energy, maca flour, gelatinization of maca.

\section{INTRODUCCIÓN}

La maca (Lepidum peruvianum Chacón o Lepidium meyenii Walpers) es una planta herbácea de la familia Brassicaceae que crece en la región andina del centro del Perú, por encima de los $3500 \mathrm{~m}$ sobre el nivel del mar. Ha sido cultivada y usada con fines alimenticios y

\footnotetext{
${ }^{a}$ Facultad de Ingeniería Industrial y de Sistemas, Universidad Nacional Federico Villarreal, Av.

Colonial 450, Lima 01, Perú.

vromero@unfv.edu.pe

b Instituto de Quimica-Fisica “Rocasolano”, CSIC, Serrano 119, 28006, Madrid, España.
} 
medicinales desde épocas pre-colombinas y en la actualidad es ampliamente promocionada especialmente como suplemento dietético con funciones multi-farmacológicas ${ }^{1}$.

Se cultivan más de ocho ecotipos diferentes de maca, los que se distinguen de acuerdo con el color de sus raíces ${ }^{1,2}$, siendo el amarillo, rojo y negro los principales y de ellos, el amarillo el ecotipo más común. La raíz-tubérculo (hipocotilo) de la maca es comestible y alimento importante para los muchos pueblos indígenas de los andes peruanos. Los hipocotilos de maca se comen frescos o pueden secarse y almacenarse para su posterior consumo. Las raíces secas se pueden comer después de hervir en agua o leche, o convertidos en jugos, cócteles, bebidas alcohólicas o café maca. En la medicina tradicional destaca su uso como un afrodisíaco para mejorar el deseo sexual y la fertilidad tanto en seres humanos como en animales.

La composición de la maca ha sido analizada y elucidada por varios grupos de investigación ${ }^{3,4}$, usando una serie de técnicas analíticas. Teniendo en cuenta estos resultados, podemos afirmar que la maca es abundante en proteínas, ácidos grasos insaturados y minerales. Las raíces de maca fresca pueden tener más de $80 \%$ de contenido de agua; su polvo deshidratado contiene entre 8,87 y $11,6 \%$ de proteína, 1,9 y $2,2 \%$ de lípidos, 54,6 y $60,0 \%$ de hidratos de carbono (23,4\% de sacarosa, $1,55 \%$ de glucosa, 4,56\% oligosacáridos, 30,4\% polisacáridos), 8,23 y $9,08 \%$ de fibra, 4,9 y 5,0\% de cenizas. Hay 18 o 19 tipos de aminoácidos en la raíz de maca. Entre ellos se encuentran siete aminoácidos esenciales (aunque no fue detectado el triptófano), y su contenido (342,6 a $388,6 \mathrm{mg} / \mathrm{g}$ proteína) es más alto que en la papa o zanahoria. El contenido de ácidos grasos insaturados, tales como ácido linoleico y oleico, es de 52,7 a 60,3\% del total de ácidos grasos. El polvo de raíz de maca es también abundante en minerales. El contenido de metales, reportado por Dini et al.3 fue, en mg por cada 100 mg de maca seca: $16,6 \mathrm{Fe}, 0,8 \mathrm{Mn}, 5,9 \mathrm{Cu}, 3,8 \mathrm{Zn}, 18,7 \mathrm{Na}, 150 \mathrm{Ca}$ y $2050 \mathrm{~K}$. La presencia de metales nocivos como $\mathrm{Pb}$ y $\mathrm{Cd}$, en muy bajas concentraciones, fueron reportados por $\mathrm{S}$. Ju et al. ${ }^{5}$

En las últimas dos décadas su interés se ha incrementado debido al estudio de sus propiedades medicinales que hacen de la maca un excelente producto del mercado nutracéutico; consecuencia de ello, las exportaciones peruanas de maca seca y procesada aumentaron casi cuatro veces en la última década, siendo más de 10 millones de dólares en $2013^{6}$. Se han reportado efectos benéficos de la maca para la salud, con estudios sobre todo en ratones, que cubren el tratamiento de una amplia gama de enfermedades tales como las disfunciones sexuales, hiperplasia prostática benigna ${ }^{7} \mathrm{u}_{\text {osteoporosis }}{ }^{8}$; asimismo se han reportado estudios clínicos que dan cuenta del incremento de la fertilidad ${ }^{9}$, la memoria y el aprendizaje $\mathrm{e}^{10,11}$, de su actividad estrogénica ${ }^{12}$, sus funciones anti-proliferativas (cáncer) y anti-inflamatorias ${ }^{13}$, anti-patógenas, anti-virales ${ }^{14}$, anti-oxidativas ${ }^{15}$, energizantes ${ }^{16}$ y protectoras (de la piel frente a radiación UV). Los efectos biológicos ejercidos por el consumo de la maca se atribuyen a la presencia de compuestos bioactivos, tales como los novedosos ácidos grasos poliinsaturados (macaenes, 0.09-0,45\%) y sus amidas (macamidas o $\mathrm{N}$-benzylamidas, 0,06$0,52 \%$ ) involucrados en funciones sexuales y que no están presentes en otras plantas ${ }^{17-20}$; glucosinolatos $^{21}$, alcaloides (lepidilinas y macaridinas) ${ }^{22}$, fitosteroles (estigmasterol, campesterol o $\beta$-sitosterol) y otros compuestos fenólicos. 
La maca es, pues, un producto que despierta considerable interés a nivel mundial, por lo que está sujeta a un creciente estudio; sin embargo muchas de sus propiedades químico-físicas y la acción de las mismas, se desconocen, no están debidamente cuantificadas o en general no están suficientemente esclarecidas.

En este contexto, no tenemos constancia de que grupo alguno de investigación haya determinado experimentalmente y de manera directa y precisa valores caloríficos de productos de la maca. En este trabajo determinamos, entre otras propiedades, la energía másica de la harina de maca, utilizando calorimetría de combustión.

La calorimetría de combustión es considerada como el método experimental más adecuado para la determinación directa y precisa de entalpías de formación $\left(\Delta H_{f}^{o}\right)$ de compuestos orgánicos y también energías másicas o valores caloríficos $(q)$ de biomasa o productos orgánicos como la maca. En el proceso de combustión en atmósfera de $\mathrm{O}_{2}$ (ecuación 1), el producto orgánico sufre la total ruptura de su esqueleto carbonado con desprendimiento de la energía contenida en los enlaces de sus moléculas:

$$
\text { Harina de maca }+\mathrm{O}_{2} \rightarrow \mathrm{CO}_{\mathrm{x}}+\mathrm{H}_{2} \mathrm{O}+\left(\mathrm{NO}_{\mathrm{x}}+\mathrm{SO}_{\mathrm{x}}+\text { etc }\right)
$$

La energía de combustión liberada produce incrementos de temperatura en el calorímetro, que se registran en función del tiempo que dura el experimento. Los calorímetros de combustión de alta precisión permiten determinar la energía de combustión del producto estudiado con una precisión inferior a $0,02 \%{ }^{23}$.

\section{PARTE EXPERIMENTAL}

\section{Muestras de harina de maca}

El producto de partida consistió de $250 \mathrm{~g}$ de harina de maca, ecotipo amarillo, suministrada por la empresa Productos Naturales "Mi Tierra" (Lima-Perú). El contenido de agua de este producto, $3,9 \%$ del peso total, fue determinado como la pérdida de peso de aproximadamente $100 \mathrm{~g}$ de muestra secada en una campana de vacío $\left(10^{-2} \mathrm{mBar}\right)$, a temperatura ambiente y durante 8 horas. Asumimos que el material evacuado fue básicamente agua.

Todos los experimentos reportados en este trabajo se realizaron utilizando harina de maca seca, obtenida mediante el procedimiento antes descrito, y después tamizada con una malla de 0,147 mesh. Las muestras convenientemente preparadas fueron pesadas en una balanza Metler AT 20 con precisión de $\pm 1 \mu \mathrm{g}$.

\section{Calorimetría de combustión}

Los experimentos de combustión fueron realizados en un calorímetro del tipo isoperibol equipado con un baño termostatizado $\left(T_{\text {baño }}=25,43 \pm 0,01^{\circ} \mathrm{C}\right)$ y una bomba de combustión estática $^{23,24}$. La bomba de combustión $(0,38 \mathrm{~L})$ incluye un dispositivo eléctrico de ignición 
con mecha de algodón. El calorímetro propiamente dicho es una vasija de 3,5 L llena de agua, en cuyo interior se sitúa: i/ la bomba de combustión, ii/ un dispositivo de agitación y iii/ un termómetro de resistencia de $\operatorname{Pt}\left(25 \Omega\right.$ de alta precisión, $\pm 10^{-4}{ }^{\circ} \mathrm{C}$, el cual va unido a un puente alterno de resistencias (Model F26, Automatic System Laboratories).

Antes y después de un experimento de combustión se realizaron mediciones y controles con todos los componentes del proceso de combustión: a) la harina de maca seca es previamente prensada en forma de pastillas de entre 512 y $531 \mathrm{mg}$ de masa, b) llenado de la bomba con $\mathrm{O}_{2}$ puro hasta alcanzar una presión de $30 \mathrm{~atm}$, c) los productos finales condensados son removidos con agua Milli-Q y caracterizados con una valoración de acidez total, mediante disolución estándar de $0,1 \mathrm{~N} \mathrm{NaOH}$. La bomba incluye $1 \mathrm{~mL}$ de agua en su interior para facilitar la disolución ácida de productos finales, que en el caso de materia orgánica suelen ser gases como $\mathrm{CO}_{\mathrm{x}}, \mathrm{NO}_{\mathrm{x}}, \mathrm{SO}_{\mathrm{x}}$; los dos últimos condensan a $\mathrm{HNO}_{3}$ y $\mathrm{H}_{2} \mathrm{SO}_{4}$. La eventual presencia de gases residuales como $\mathrm{SO}_{2} \mathrm{y} \mathrm{Cl}_{2}$ se cuantifican mediante tubos Dragër (precisión de $\pm 10-15 \%$ ), iv/ aseguramos que la combustión sea completa (usando igualmente tubos Dragër), esto es que todo el carbono de la materia orgánica quemada pase a $\mathrm{CO}_{2}$. Para facilitar tal proceso utilizamos ácido benzoico como fundente auxiliar, prensada también en forma de pastillas de entre 261 y $274 \mathrm{mg}$ de masa.

Del registro temperatura vs tiempo ( $T$ vs t) de un experimento de combustión que dura $1 \mathrm{~h}$, obtenemos valores que permiten cuantificar el incremento de temperatura adiabático $\Delta T_{a d}$ asociado al proceso estudiado.

Es imprescindible calibrar el calorímetro; para lo cual utilizamos la combustión del ácido benzoico (sustancia de referencia) cuya energía másica de combustión ha sido determinada con precisión bajo condiciones específicas certificadas por el $\operatorname{NIST}^{25}\left(q_{\text {benz }}=26634 \pm 3 \mathrm{~J} / \mathrm{g}\right)$. El parámetro de calibrado عo, denominado equivalente energético se define como sigue:

$$
\varepsilon_{\mathrm{o}}=\left(\mathrm{m}_{\mathrm{benz}} \bullet q_{\text {benz }} / \Delta T_{a d}\right)-\varepsilon^{\mathrm{i}}
$$

donde mbenz, es la masa del ácido benzoico quemado, $\varepsilon^{i}$ es la suma de las capacidades caloríficas de los elementos que contiene la bomba en el estado inicial; $\Delta T_{a d}$ es el incremento de temperatura adiabático. A partir de 5 experimentos obtuvimos un valor promedio de $\varepsilon_{\mathrm{o}}=$ $\mathbf{1 4 2 4 9 , 6} \pm \mathbf{5 , 2} \mathrm{J} /{ }^{\circ} \mathrm{C}$; donde la incertidumbre consignada es la incertidumbre estándar del valor promedio. Una vez determinado $\varepsilon_{\mathrm{o}}$, los experimentos con la harina de maca se ajustaron lo más posible a las condiciones en las que se hizo el calibrado, eliminándose de esta manera los errores sistemáticos.

El valor calorífico (o energía másica) de la harina de maca, $q_{m}$, se obtiene a partir de la siguiente relación,

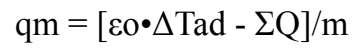

donde $\Sigma$ Q incluye energías como las de ignición, dilución de ácidos, combustión de sustancias 
auxiliares o fundentes (ácido benzoico en nuestro caso) y m es la masa del producto estudiado. Dado que las muestras han sido secadas, qm se denomina también "valor calorífico neto" $(\mathrm{NCV})^{26,27}$.

\section{Calorimetría diferencial de barrido (Differencial Scanning Calorimter, DSC)}

Para la determinación experimental de las propiedades termofísicas de la harina de maca se utilizó el Calorímetro Diferencial de Barrido ("Differential Scanning Calorimetry", DSC), Perkin Elmer Pyris 1 con sistema criogénico cerrado (intra-cooler) en el rango de $-20^{\circ} \mathrm{C}$ a $450^{\circ} \mathrm{C}$ y funcionando bajo atmósfera de $\mathrm{N}_{2}$. Para el calibrado del equipo se utilizó indio metálico como material específico de referencia, cuya temperatura y entalpía de fusión son respectivamente, $T_{\text {fus }}=161^{\circ} \mathrm{C}$ y $\Delta H_{\text {fus }}=28,8 \mathrm{~J} / \mathrm{g}$.

Tanto para la determinación del calor específico en función de la temperatura, c.e.( $(T)$, como para la degradación o descomposición de la harina de maca se usaron cápsulas de aluminio. Utilizamos entre 2 y $4 \mathrm{mg}$ de masa por muestra encapsulada y los termogramas DSC se registraron a una velocidad de barrido de $10{ }^{\circ} \mathrm{C} / \mathrm{min}$. Los valores de calor específico se determinaron teniendo como referencia los valores conocidos del calor específico c.e. $(T)$ del zafiro. Muestra y zafiro fueron registrados con la misma cápsula.

Para la determinación de parámetros de gelatinización se usaron cápsulas de acero inoxidable, con volumen máximo de $30 \mu \mathrm{L}$, y tapas recubiertas de oro. A la harina de maca (entre 5 y $7 \mathrm{mg}$ ) se le agregó agua, aproximadamente el triple de la masa consignada (15 a $21 \mu \mathrm{L})$. Las cápsulas incluyendo las mezclas agua-harina fueron selladas herméticamente y después mantenidas en un soporte de balanceo para su homogeneización, durante al menos 12 horas. Los termogramas DSC se registraron en un rango de temperaturas de 10 a $120^{\circ} \mathrm{C}$ y velocidad de barrido fue de $10{ }^{\circ} \mathrm{C} / \mathrm{min}$.

\section{RESULTADOS Y DISCUSIÓN}

\section{Determinación de la energía másica o el valor calorífico neto}

Los resultados experimentales obtenidos de la combustión de la harina de maca se consignan en la tabla 1.

El valor promedio de la energía másica o valor calorífico neto de la harina de maca seca, $<q_{\text {maca }}>$, determinado es:

$<q_{\text {maca }}>=17512 \pm 135 \mathrm{~J} / \mathrm{g}=1751 \pm 14 \mathrm{~kJ} / 100 \mathrm{~g}=419 \pm 3 \mathrm{Kcal} / 100 \mathrm{~g}$

la incertidumbre de la medida representa el $0,8 \%$ del valor promedio encontrado. El valor que determinamos es más del doble que el indicado por Mc Neil et al.1 como "energy content" (663 kJ/100g), el cual es sólo una estimación aproximada al igual que muchos de los valores reportados en páginas webb. Nuestro valor es casi $18 \mathrm{Kcal} / 100 \mathrm{~g}$ más bajo que el que determinamos para la quinua o la kiwicha ${ }^{28}$.

A partir de los datos de valoración de disolución ácida de productos finales de la combustión, 
encontramos que esta disolución tiene, en promedio, una molaridad de $42 \mathrm{mM}$; la cual creemos estaría formada principalmente por $\mathrm{HNO}_{3}$, dado que en el control de gases residuales realizados con indicadores Dragüer no detectamos indicios de $\mathrm{SO}_{\mathrm{x}}$ ni de $\mathrm{Cl}_{2}(<1 \mathrm{ppm})$.

Tabla 1. Resultados de la combustión de la harina de maca secada al vacío

\begin{tabular}{|c|c|c|c|c|}
\hline & Exp. 1 & Exp. 2 & Exp. 3 & Exp. 4 \\
\hline Harina de maca (mg) & 512,44 & 513,71 & 530,22 & 529,53 \\
\hline Masa Ac. Benzoico (mg) & 262,58 & 261,85 & 273,91 & 264,76 \\
\hline Masa algodón (mg) & 3,95 & 2,92 & 2,75 & 2,72 \\
\hline$\Delta T_{\mathrm{ad}}\left({ }^{\circ} \mathrm{C}\right)$ & 1,1355 & 1,1214 & 1,1563 & 1,1380 \\
\hline Vol. NaOH 0,1N (mL) & 0,40 & 0,45 & 0,38 & 0,45 \\
\hline $\mathrm{Q}_{\text {benzoico }}(\mathrm{J})$ & 6940,9 & 6921,6 & 7240,5 & 6998,7 \\
\hline $\mathrm{Q}_{\text {algodón }}(\mathrm{J})^{(\mathrm{a})}$ & 68,7 & 50,8 & 47,8 & 47,3 \\
\hline $\mathrm{Q}_{\text {Ac. nitrico }}(\mathrm{J})^{(\mathrm{b})}$ & 2,4 & 2,7 & 2,3 & 2,7 \\
\hline $\mathrm{Q}_{\text {ignición }}(\mathrm{J})^{(\mathrm{c})}$ & 0,8 & 0,8 & 0,8 & 0,8 \\
\hline $\mathrm{Q}_{\text {maca }}(\mathrm{J})$ & 9167,3 & 9003,2 & 9184,7 & 9166,3 \\
\hline$q_{\text {maca }}(\mathrm{J} / \mathrm{g})$ & 17889,5 & 17252,9 & 17322,6 & 17310,2 \\
\hline$<q_{\text {maca }}>(\mathrm{J} / \mathrm{g})^{(\mathrm{d})}$ & \multicolumn{4}{|c|}{$17512 \pm 135$} \\
\hline$<q_{\text {maca }}>(\mathrm{kJ} / 100 \mathrm{~g})$ & \multicolumn{4}{|c|}{$1751 \pm 14$} \\
\hline$<q_{\text {maca }}>(\mathrm{Kcal} / 100 \mathrm{~g})^{(\mathrm{e})}$ & \multicolumn{4}{|c|}{$419 \pm 3$} \\
\hline
\end{tabular}

(a) Energía de la mecha de algodón obtenida considerando el valor $q_{\text {algodón }}=17409,8$ $\mathrm{J} / \mathrm{g}$, determinado en nuestro laboratorio; ${ }^{(b)}$ Energía obtenida a partir de la valoración ácida y teniendo en cuenta la energía de formación del $\mathrm{HNO}_{3}{ }^{\left({ }^{(c)}\right.}$ Energía de ignición por descarga de un condensador a $18 \mathrm{~V}$. (d) Valor promedio e incertidumbre estándar;

(e) Valor promedio en Kcal por $100 \mathrm{~g}$ de muestra.

\section{Determinación de calores específicos}

Los valores de calor específico, c.e. (en J/g. ${ }^{\circ} \mathrm{C}$ ), representados en la figura 1 son una media de 4 medidas realizadas en el rango de -4 a $70^{\circ} \mathrm{C}$. Como se puede apreciar entre 14 y $21{ }^{\circ} \mathrm{C}$ hay una variación en la tendencia creciente de c.e. $(T)$, resultado que podemos atribuir a cambios estructurales del tipo orden-desorden. El c.e. a $25^{\circ} \mathrm{C}$ es igual a $1,46 \pm 0,03 \mathrm{~J} / \mathrm{g} .{ }^{\circ} \mathrm{C}$; valor comparable al correspondiente c.e. del trigo, con humedad relativa entre el 0 y el 6,23\%, reportado por Y. Cao et al..$^{29}$

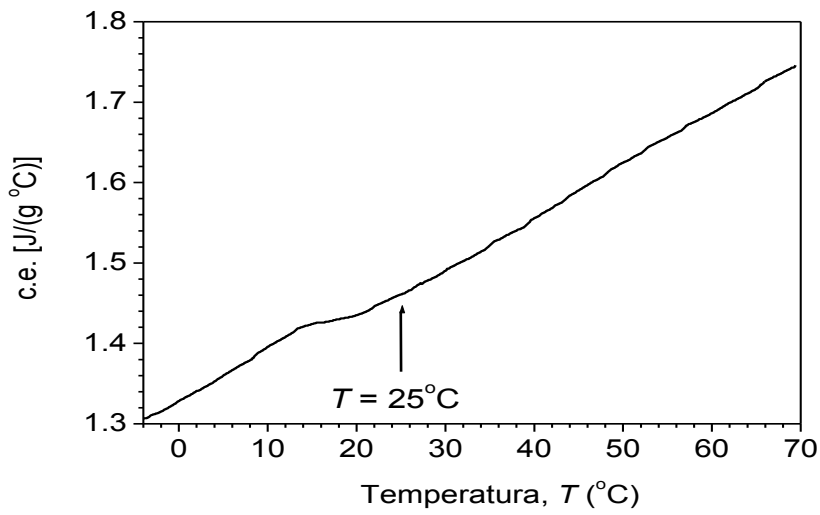

Figura 1. Curva de calores específicos c.e. en función de la temperatura, $T$ 


\section{Descomposición térmica de la harina de maca}

Los termogramas DSC registrados entre 50 y $230{ }^{\circ} \mathrm{C}$ (figura 2 ) muestran picos endotérmicos y asimétricos muy intensos con un máximo en $T_{\max }=210,1 \pm 0,4^{\circ} \mathrm{C}$ y entalpía igual a $\Delta H_{d e c}$ $=82 \pm 10 \mathrm{~J} / \mathrm{g}$, relacionados con la completa degradación y descomposición de la maca. Estos valores son aproximadamente $7^{\circ} \mathrm{C}$ y $20 \mathrm{~J} / \mathrm{g}$ más bajos que los correspondientes a la descomposición de la cañihua seca ${ }^{30}$.

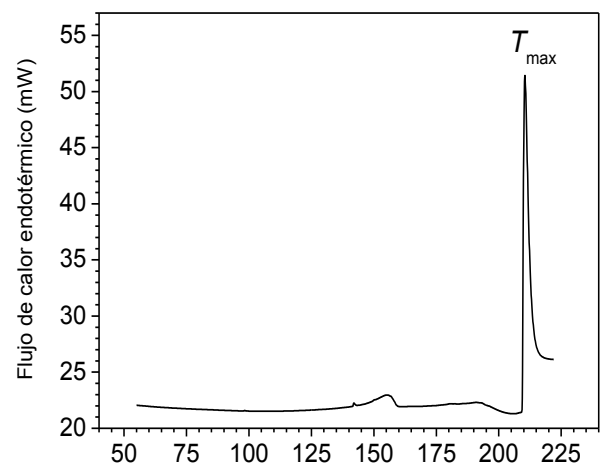

Figura 2. Termograma DSC típico mostrando un pico intenso de la descomposición de la harina de maca

\section{Proceso de gelatinización}

Es importante mencionar que la gelatinización es un proceso asociado con el calentamiento en agua del almidón (polímero de glucosas con arreglo semicristalino incluido dentro de gránulos) de la maca y está relacionado con la pérdida de su ordenamiento molecular ${ }^{31}$. El paso del ordenamiento semi-cristalino del almidón (no digerible), a una forma eventualmente amorfa (digerible) va acompañada de cambios estructurales (varios irreversibles) como: absorción de agua, hinchazón del grano, fusión de la parte cristalina, pérdida de la birrefringencia, aumento en la viscosidad y la solubilidad del gránulo, entre otros cambios.

Los termogramas DSC, como el de la figura 3, muestran picos “anchos" entre 70 y $85{ }^{\circ} \mathrm{C}$ atribuibles al proceso de gelatinización. En la tabla 2 se consignan valores promedio, de 4 medidas, de la entalpía y las temperaturas asociadas con este proceso.

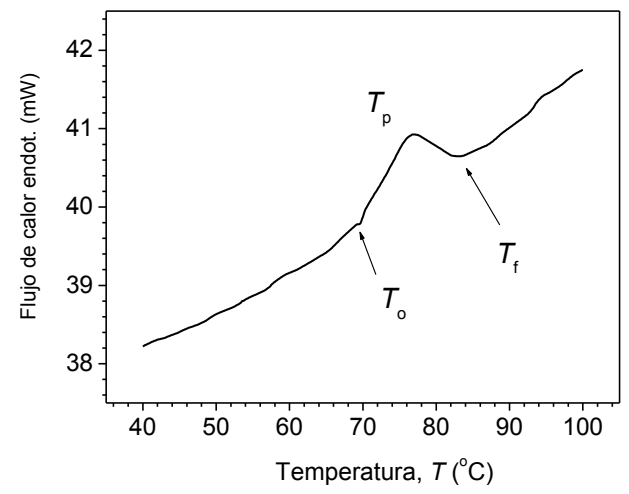

Figura 3. Termograma DSC típico del proceso de gelatinización de la maca 
Tabla 2. Parámetros termofísicos del proceso de gelatinización de la harina de maca

\begin{tabular}{cccc}
\hline $\begin{array}{c}T_{\mathrm{o}}\left({ }^{\circ} \mathrm{C}\right) \\
\begin{array}{c}\text { Temperatura } \\
\text { inicial }\end{array}\end{array}$ & $\begin{array}{c}T_{\mathrm{p}}\left({ }^{\circ} \mathrm{C}\right) \\
\text { Temperatura del pico } \\
\text { de gelatinación }\end{array}$ & $\begin{array}{c}T_{\mathrm{f}}\left({ }^{\circ} \mathrm{C}\right) \\
\text { Temperatura final }\end{array}$ & $\begin{array}{c}\Delta H_{\text {gel }}(\mathrm{J} / \mathrm{g}) \\
\text { entalpía de } \\
\text { gelatinización }\end{array}$ \\
\hline $70,0 \pm 0,4$ & $76,9 \pm 0,4$ & $83,4 \pm 0,6$ & $5,6 \pm 0,5^{\mathrm{a}}$ \\
\hline${ }^{\mathrm{a}}$ Calculado considerando sólo la masa de la harina seca &
\end{tabular}

La entalpía de gelatinización $\Delta H_{\text {gel }}$ que reportamos es comparable a la determinada por Finardi-Filho et al. ${ }^{32}(6,22 \mathrm{~J} / \mathrm{g})$ para el almidón extraído de la maca; mas no así la temperatura de gelatinización $T_{p}$ que en nuestro caso es casi $29{ }^{\circ} \mathrm{C}$ más alto que la reportada por ellos. Para explicar esta diferencia hay que tener en cuenta que los parámetros de gelatinización determinados por Finardi-Filho et al. corresponden al almidón aislado de la maca. Sin embargo, no descartamos diferencias en concentración de componentes y/o en las estructuras moleculares del almidón estudiado por los investigadores antes mencionados y el contenido en nuestra harina de maca; dado que habitualmente se asocian elevadas temperaturas de gelatinización $T_{p}$ con elevados contenidos de amilosa, respecto de amilopectina, aunque otros estudios revelan que las propiedades de gelatinización reflejan más bien la arquitectura molecular de las amilopectinas (abundancia de unidades de canal cortos para $T_{p}$ y $\Delta H_{\text {gel }}$ bajos) y no de la relación amilosa/amilopectina ${ }^{33,34}$.

La $T_{p}$ de la harina de maca determinada es comparable a la del maíz ${ }^{35}$, camote ("sweet potato") ${ }^{36}$ o de la yuca ("cassava") ${ }^{36,37}$, pero no la $\Delta H_{\text {gel }}$ que es entre 4 y $5 \mathrm{~J} / \mathrm{g}$ más baja que la de estos productos. ${ }^{32-36}$

\section{CONCLUSIONES}

Estudiamos propiedades termoquímicas y termofísicas de la harina de maca (del ecotipo amarillo) comercial, previamente tamizada y secada al vacío. Utilizamos la calorimetría de combustión para determinar, creemos por primera vez, el valor calorífico neto o energía másica de combustión igual a $17512 \pm 135 \mathrm{~J} / \mathrm{g}$ ó $419 \pm 3 \mathrm{Kcal} / 100 \mathrm{~g}$. Asimismo, utilizamos calorimetría diferencial de barrido (DSC) para evaluar, i/el calor específico c.e. en rango de -4 a $70 \mathrm{oC}$, siendo c.e. a $25 \mathrm{oC}$ igual a $1,46 \pm 0,03 \mathrm{~J} / \mathrm{g} . \mathrm{oC}$; ii/ la temperatura y entalpía del proceso de descomposición iguales a, respectivamente, $210,1 \pm 0,4$ oC y $82 \pm 10 \mathrm{~J} / \mathrm{g}$; iii/ parámetros termofísicos de la gelatinización como la temperatura y entalpía iguales a, respectivamente, $76,9 \pm 0,4$ oC y $5,65,6 \pm 0,5 \mathrm{~J} / \mathrm{g}$.

\section{AGRADECIMIENTO}

JZDP agradece al Programa PNICP (INNÓVATE PERÚ, ECIP-1-P-030-14) "Estancias cortas de investigadores peruanos residentes en el extranjero". 


\section{REFERENCIAS BIBLIOGRÁFICAS}

1. Wang Y, Wang Y, McNeil B, Harvey LM. Maca: An Andean crop with multipharmacological functions. Food Res Int. 2007; 40(7):783-92.

2. Gonzales C, Rubio J, Gasco M, Nieto J, Yucra S, Gonzales GF. Effect of short-term and long-term treatments with three ecotypes of Lepidium meyenii (MACA) on spermatogenesis in rats. J Ethnopharmacol. 2006; 103(3):448-54.

3. Dini A, Migliuolo G, Rastrelli L, Saturnino P, Schettino O. Chemical composition of Lepidium meyenii. Food Chem. 1994; 49(4): 347-9.

4. Yu LJ, Jin WW. Study on the nutritional components and the anti-fatigue effects of dry powder of maca (Lepidium meyenii). Food Sci. 2004; 25(2):164-166.

5. Ju S, Yu J, Ma Y, Yang Y, Liu M. Rapid Determination of Cadmium and Lead in Maca (Lepidium meyenii) by Magnetic Solid-Phase Extraction and Flame Atomic Absorption Spectrometry. Anal Lett. 2015; 48(16):2566-80.

6. PROMPERU. Evolución de las exportaciones del producto: Maca. [En línea]. Perú; 2013. [consultado el 18 de setiembre del 2014]. Disponible en: http://www.siicex.gob. $\mathrm{pe} /$ siicex/apb/ReporteResumen.aspx?psector=1025\&preporte=prodresu\&pvalor=1934.

7. Gasco M, Villegas L, Yucra S, Rubio J, Gonzales GF. Dose-response effect of Red Maca (Lepidium meyenii) on benign prostatic hyperplasia induced by testosterone enanthate. Phytomedicine. 2007; 14(7-8):460-4.

8. Liu H, Jin W, Fu C, Dai P, Yu Y, Huo Q, et al. Discovering anti-osteoporosis constituents of maca (Lepidium meyenii) by combined virtual screening and activity verification. Food Res Int. 2015; 77, Part 2:215-20.

9. Gonzales GF, Ruiz A, Gonzales C, Villegas L, Cordova A. Effect of Lepidium meyenii (Maca) roots on spermatogenesis of male rats. Asian J Androl. 2001; 3: 231-233.

10. López-Fando A, Gómez-Serranillos MP, Iglesias I, Lock O, Upamayta UP, Carretero ME. Lepidium peruvianum chacon restores homeostasis impaired by restraint stress. Phytother Res. 2004; 18(6):471-4.

11. Rubio J, Qiong W, Liu X, Jiang Z, Dang H, Chen SL, Gonzales GF. Aqueous Extract of Black Maca (Lepidium meyenii) on Memory Impairment Induced by Ovariectomy in Mice. Evid Based Complement Alternat Med. 2011; 2011:253958.

12. Valentová K, Buckiová D, Kren V, Peknicová J, Ulrichová J, Simánek V. The in vitro biological activity of Lepidium meyenii extracts. Cell Biol Toxicol. 2006; 22(2):91-9.

13. Bai N, He K, Roller M, Lai CS, Bai L, Pan MH. Flavonolignans and other constituents from Lepidium meyenii with activities in anti-inflammation and human cancer cell lines. J Agric Food Chem. 2015; 63(9):2458-63.

14. Del Valle Mendoza J, Pumarola T, Gonzales LA, Del Valle LJ. Antiviral activity of maca (Lepidium meyenii) against human influenza virus. Asian Pac J Trop Med. 2014; 7S1: S415-20.

15. Puoci F, Malanchin R, Piangiolino C, Restuccia D, Curcio C, Parisi OI, et al. Maca flour: a powerful ingredient for functionally enhanced bread. Int Food Res J. 2013; 20(3):1293-1300.

16. Zheng BL, He K, Hwang ZY, Lu Y, Yan SJ, Kim CH, et al. Effect of aqueous extract from Lepidium meyenii on mouse behavior in forced swimming test. En Ho CT, Zheng 
QY. Eds. Quality Management of Nutraceuticals. Washington DC: American Chemical Society; 2002. p. 90-100.

17. Ganzera M, Zhao J, Muhammad I, Khan IA. Chemical profiling and standardization of Lepidium meyenii (Maca) by reversed phase high performance liquid chromatography. Chem Pharm Bull (Tokyo). 2002; 50(7):988-91.

18. McCollom MM, Villinski JR, McPhail KL, Craker LE, Gafner S. Analysis of macamides in samples of Maca (Lepidium meyenii) by HPLC-UV-MS/MS. Phytochem Anal. 2005;16(6):463-9.

19. Wu H, Kelley CJ, Pino-Figueroa A, Vu HD, Maher TJ. Macamides and their synthetic analogs: evaluation of in vitro FAAH inhibition. Bioorg Med Chem. 2013; 21(17):518897.

20. Esparza E, Hadzich A, Kofer W, Mithöfer A, Cosio EG. Bioactive maca (Lepidium meyenii) alkamides are a result of traditional Andean postharvest drying practices. Phytochemistry. 2015; 116:138-48.

21. Campos D, Chirinos R, Barreto O, Noratto G, Pedreschi R. Optimized methodology for the simultaneous extraction of glucosinolates, phenolic compounds and antioxidant activity from maca (Lepidium meyenii). Ind Crops Prod. 2013; 49: 747-754.

22. Muhammad I, Zhao J, Dunbar DC, Khan IA. Constituents of Lepidium meyenii 'maca'. Phytochemistry. 2002; 59(1):105-10.

23. Dávalos JZ, Roux MV, Jiménez P. Calorímetro de combustión para compuestos orgánicos. REVCIUNI. 2000; 6 (1): 92.

24. Dávalos JZ, Roux MV, Jiménez P. Evaluation of Poultry litter as a feasible Fuel. Thermochim Acta. 2002; 394: 261-266.

25. NIST Chemistry Webbook. NIST Standard Reference Database Number 69 [En línea]. Gaithersburg MD: Linstrom PJ, W.G. Mallard WG (Eds.); National Institute of Standards and Technology; 2014. [consultado el 7 de MAYO del 2014]. Disponible en: http://www.webbok.nist.gov

26. Toscano G, Pedretti EF. Calorific value determination of solid biomass fuel by simplified method. J of Ag Eng. - Riv di Ing Agr. 2009; 3:1-6.

27. Gravalos I, Kateris D, Xyradakis P, Gialamas T, Loutridis S, Augousti A, et al. FORMEC 2010, Forest Engineering: Meeting the Needs of the Society and the Environment, Padova- Italy, 2010.

28. Dávalos J, A. Tirado A, V. Romero V, en preparación.

29. Cao Y, Li G, Zhang Z, Chen L, Li Y, Zhang T. The specific heat of wheat. 10th International Working Conference on Stored Product Protection. Julius-Kühn-Archiv. 2010; 425: 243-249.

30. Romero V, A. Tirado A, J. Dávalos J. Determinación experimental mediante DSC de las estabilidades térmicas y las capacidades caloríficas: quinua, kiwicha y cañihua. Cátedra Villarreal. 2015; 3(1): 47-52.

31. Atwell WA, Hood LF, Lineback DR, Varriano-Marston E, Zobel HF. The terminology and methodology associated with basic starch phenomena. Cereal Foods World. 1988; 33: 306-311.

32. Rondán-Sanabria, F. Finardi-Filho F. Physical-chemical and functional properties of maca root starch (Lepidium meyenii Walpers). Food Chem. 2009; 114(2): 492-498. 
33. Noda T, Takahata Y, Sato T, Suda I, Morishita T, Ishiguro K, et al. Relation-ships between chain length distribution of amylopectin and gelatinization properties within the same botanical origin for sweet potato and buckwheat. Carbohydr Polym. 1998; 37: 153-158.

34. Noda T, Kimura T, Otani M, Ideta O, Shimada T, Saito A, et al. Physicochemical properties of amylose-free starch from transgenic seet potato. Carbohydr Polym. 2002; 49: 253-260.

35. Osundahunsi FO, Fagbemi NT, Kesselman E, Simón E. Comparison of the physicochemical properties and pasting characteristics of flour and starch from red and white sweet potato. J Agricultural Food Chem. 2003; 51: 2232-2236.

36. Pérez EE, Breene WM, Bahnassey YA. Variations in the Gelatinization Profiles of Cassava, Sagu and Arrowroot Native Starches as Measured with Different Thermal and Mechanical Methods. Starch/Stärke. 1998; 50: 70-72. 